\title{
Making matters worse for a broken heart
}

\author{
Norbert Frey and Eric N. Olson
}

Department of Molecular Biology, University of Texas Southwestern Medical Center at Dallas, Dallas, Texas, USA

Address correspondence to: Eric N. Olson, Department of Molecular Biology, University of Texas Southwestern Medical Center at Dallas, 6000 Harry Hines Boulevard, Dallas, Texas 75390-9148, USA. Phone: (214) 648-1187; Fax: (214) 648-1196; E-mail: eolson@hamon.swmed.edu.

Familial hypertrophic cardiomyopathy (FHC) is an autosomal-dominant inherited disease characterized by increased cardiac mass, arrhythmias, and sudden death. It has been estimated that as many as 1 in 500 people carry a mutation in a sarcomeric protein gene that can result in FHC (1). Myofilaments containing mutant sarcomeric proteins have been shown to exhibit alterations in calcium sensitivity, myofilament velocity, myosin ATPase activity, and maximum force development (reviewed in ref. 2). However, it remains unclear how these changes in the contractile apparatus transduce signals that result in hypertrophy and reprogramming of cardiac gene expression.

There have been numerous attempts to model human sarcomeric mutations in animals (3), using either transgenesis to misexpress mutant proteins or gene replacement to introduce mutations into the mouse genome to mimic specific human mutations. The first example of the latter approach involved the introduction of a glutamine for arginine residue at codon 403 of $\alpha$ myosin heavy chain $(\alpha \mathrm{MHC})$, which is analogous to an especially severe FHC mutation in the human $\beta M H C$ gene (4). In humans, there is a switch from expression of $\alpha$ to $\beta \mathrm{MHC}$ after birth, such that $\beta \mathrm{MHC}$ becomes the predominant $\mathrm{MHC}$ isoform expressed in the adult heart. In mice, these isoforms are regulated oppositely, with $\alpha \mathrm{MHC}$ being the major adult isoform (5), which is why the mouse mutation was introduced into the $\alpha M H C$ gene.

Amino acid 403 is contained in the MHC head and lies within the actinbinding region. In vitro studies indicate that the R403Q mutation impairs the myosin-actin interaction (6), leading to a hypocontractile state which, in turn, may trigger compensatory car- diac hypertrophy. However, in contrast to the severity of the corresponding human $\beta \mathrm{MHC}$ mutation, the mouse $\alpha \mathrm{MHC}$ mutation results in an extremely mild hypertrophic response without associated lethality.

\section{Calcium signaling and sarcomere mutations}

Recent attention has focused on the possibility that the calcium/calmodulin-dependent protein phosphatase calcineurin transduces hypertrophic signals from the sarcomere, a notion supported by several lines of evidence. First, activated calcineurin expressed under control of the $\alpha M H C$ promoter can evoke a profound hypertrophic response in vivo, as well as in cultured primary cardiomyocytes (7). Second, the calcineurin inhibitors cyclosporin A (CsA) and FK506 can block cardiomyocyte hypertrophy and can rescue several rodent models of this disorder, including mice with sarcomeric dys-

surgically (12) induced hypertrophy, as well as in failing human hearts (13).

In an article in the previous issue of the JCI, Fatkin et al. (14) treated mice heterozygous for the $\alpha M H C^{403}$ mutation with CsA and FK506. Remarkably, both agents severely exacerbated the cardiac disease in these animals, as indicated by a more than $50 \%$ increase in ventricular wall thickness, enhanced myofibrillar disarray, and reduced survival. Treatment of these mice with the potassium channel opener minoxidil, a drug that has been shown to induce cardiac hypertrophy in rodents despite its antihypertensive properties (15), also caused an exaggerated hypertrophic response. Pretreatment with the calcium channel blocker diltiazem, a drug frequently used for treatment of FHC patients, prevented the hypertrophic effects of minoxidil and calcineurin inhibitors. The drug-induced hypertrophic response in $\alpha M H C^{403 /+}$ mice appeared to reflect an intrinsic property of cardiomyocytes, rather than a secondary response of the heart to hemodynamic alterations, although in the case of minoxidil it would be interesting to know how plasma catecholamine levels were affected, considering that increased adrenergic tone has been postulated to be responsible for minoxidil-induced cardiac hypertrophy (16).

function due to cardiac misexpression of the actin-binding protein tropomodulin $(8,9)$ or a nonphosphorylatable form of myosin light chain-2v (8). Moreover, although there is some disagreement on this issue (reviewed in ref. 10), it appears that calcineurin inhibitors can also prevent hypertrophy in response to pressure overload, saltand renovascular-induced hypertension, and myocardial infarction. Finally, calcineurin activity is elevated in hearts of mice with genetically (11) or
Since calcium/calmodulin-dependent signaling has been shown to play a critical role in cardiac hypertrophy (reviewed in ref. 17), Fatkin et al. (14) examined whether alterations in calcium metabolism could account for the hypersensitivity of $\alpha M H C^{403 /+}$ cardiomyocytes to CsA and minoxidil. As reported previously (18), CsA raised diastolic calcium concentrations significantly in wild-type cardiomyocytes, but Fatkin et al. show that $\alpha M H C^{403 /+}$ cardiomyocytes show no such change. 
Similarly, minoxidil raised the calcium concentration in wild-type but not mutant cardiomyocytes. Conversely, blockade of L-type calcium channels by diltiazem abrogated the CsA-induced increase in diastolic calcium in wildtype cardiomyocytes.

The results of this paper (14) are striking and raise several interesting questions. Paramount among them are the mechanism underlying the severe hypertrophic response and the targets for the CsA and minoxidil effect in mutant myocytes. CsA and FK506 are exquisitely specific pharmacologic agents that associate with the binding proteins cyclophilin and FKBP12, respectively. Most of the actions of these drugs are thought to be mediated by calcineurin inhibition, suggesting that calcineurin is the primary target for their effects on the heart. Since increased calcineurin activity in transgenic mouse hearts results in massive cardiac hypertrophy (7), it seems counterintuitive that calcineurin blockers can actually provoke hypertrophy. Calcineurin activity was not measured in this study, leaving open questions as to how it might be affected in $\alpha M H C^{403 /+}$ cardiomyocytes, but raising the possibility that calcineurin expression or activity is upregulated as a compensatory response in $\alpha M H C^{403 /+}$ mice. It also remains unclear whether calcineurin activity is affected by minoxidil, which apparently had the same effect as CsA, even though as a potassium channel opener it belongs to a different class of drugs.

The effects of CsA on calcium transients and diastolic calcium concentrations in wild-type cardiomyocytes and cardiac muscle preparations (18-20) may best be explained by calcium leakage through channels such as the ryanodine receptor or the IP3 receptor. In this regard, calcineurin associates with calcium release channels and modulates their activity (21). It has also been demonstrated that CsA can block calcineurin-mediated inhibition of the Ltype calcium channel (22), suggesting a mechanism whereby CsA might elevate intracellular calcium.

\section{Some open questions}

How is calcium metabolism altered in $\alpha M H C^{403 /+}$ cardiomyoctes, and why do mutant cells fail to respond to CsA treatment with an increase in calcium concentration? One possible explanation would be that the intracellular cal- cium stores are chronically calciumdepleted, so that any stimulus for increased calcium release is ineffective. In fact, it has been shown that muscle strips from $\alpha M H C^{403 /+}$ mice exhibit a higher calcium mobilization to generate the same level of force as wild-type controls (23). For this reason, calcium release may already be enhanced, thus helping to deplete intracellular calcium stores. Whether the paradoxical hypertrophic response of $\alpha M H C^{403 /+}$ cardiomyoctes to CsA is related to this altered calcium responsiveness remains to be determined.

The results of this study (14) underscore the complexity of calcium signaling in cardiomyocytes and illustrate the gaps in our knowledge of the mechanisms responsible for cardiac dysfunction in response to contractile abnormalities. However, for several reasons, the behavior of $\alpha M \mathrm{HC}^{403 /+}$ cardiomyocytes cannot necessarily be extrapolated to that of other sarcomeric mutations, particularly in humans. First, even though this mouse model resembles the human $\beta M H C$ mutation, the phenotype in mice is significantly different with respect to the extent of cardiac hypertrophy and associated lethality. Second, different sarcomeric mutations even within the same protein can affect myofilament calcium sensitivity in opposite directions (2), suggesting that treatment with drugs that modify the intracellular calcium metabolism may not necessarily have beneficial effects in all cases. Furthermore, given the ability of CsA to rescue the phenotype of tropomodulin and myosin light chain$2 \mathrm{v}$ transgenic mice (8), alterations of the sarcomere seem to induce cardiomyopathy via disparate mechanisms, which may preclude a generalized approach to treatment, at least using therapies directed at an upstream regulator of hypertrophic signaling. Third, the established benefits of calcium channel blockade in FHC patients may not be due to direct effects on intracellular calcium metabolism, but rather may be mediated indirectly by improved hemodynamics and myocardial oxygen supply. Finally, the profound physiologic differences between hearts of humans and of mice, as highlighted by the tenfold difference in resting heart rate, point to the inevitable species differences in calcium handling.

The deleterious effects of calcineurin inhibition in $\alpha M H C^{403 /+}$ mice are unan- ticipated, in light of the ability of CsA to prevent cardiac hypertrophy and improve cardiac function in other rodent models of hypertrophic cardiomyopathy. Whether these findings reflect an oddity of the $\alpha \mathrm{MHC}^{403 /+}$ phenotype or a more generalized feature of the hypertrophic response remains to be determined. Nevertheless, the work by Fatkin et al. (14) makes it clear that it is premature to initiate treatment of FHC patients with calcineurin inhibitors until many questions about the mechanisms that link cardiac contractility with the hypertrophic growth response are answered. In the future, it will be especially interesting to further define the molecular response to CsA and minoxidil in $\alpha M H C^{403 /+}$ myocytes. It will also be of interest to determine whether the functions or expression of other critical regulators of calcium homeostasis, such as calcium/calmodulin-dependent kinase, sarcoplasmic calcium ATPase, phospholamban, or the sodi$\mathrm{um} /$ calcium exchanger, are affected by these drugs. The role of calcineurin signaling in other forms of FHC and the potential involvement of other mediators, such as mitogen-activated protein kinases, adrenergic signaling, and proapoptotic pathways, in the development of FHC also need further investigation. Until these issues are addressed, we should keep in mind the paramount principle in the treatment of any disease: "First, do no harm."

1. Maron, B.J., Gardin, J.M., Flack, J.M., Gidding, S.S., and Bild, D. 1995. Prevalence of hypertrophic cardiomyopathy in a general population of young adults. Circulation. 92:785-789.

2. Redwood, C.S., Moolman-Smook, J.C., and Watkins, H. 1999. Properties of mutant contractile proteins that cause hypertrophic cardiomyopathy. Cardiovasc. Res. 44:20-36.

3. Robbins, J. 2000. Remodeling the cardiac sarcomere using transgenesis. Annu. Rev. Physiol. 62:261-287.

4. Geisterfer-Lowrance, A.A., et al. 1996. A mouse model for hypertrophic cardiomyopathy. Science. 272:731-734

5. Weiss, A., and Leinwand, L.A. 1996. The mammalian myosin heavy chain gene family. Annu. Rev. Cell Dev. Biol. 12:417-439.

6. Cuda, G.L., Fananapazir, L., Zhu, W.S., Sellers, J.R., and Epstein, N.D. 1993. Skeletal muscle expression and abnormal function of $\beta$-myosin in hypertrophic cardiomyopathy. J. Clin. Invest. 91:2861-2865.

7. Molkentin, J.D., et al. 1998. A calcineurindependent transcriptional pathway for cardiac hypertrophy. Cell. 93:215-228.

8. Sussman, M.A., et al. 1998. Prevention of cardiac hypertrophy in mice by calcineurin inhibition. Science. 281:1690-1693.

9. Lim, H.W., et al. 2000. Reversal of cardiac hypertrophy in transgenic disease models by cal cineurin inhibition. J. Mol. Cell. Cardiol. 32:697-709. 
10. Olson, E.N., and Williams, R.S. 2000. Calcineurin signaling and muscle remodeling. Cell. 101:689-692.

11. Sussman, M.A., et al. 1999. Pathogenesis of dilated cardiomyopathy. Molecular, structural, and population analyses in tropomodulin-overexpressing transgenic mice. Am. J. Pathol. 155:2101-2113.

12. Lim, H.W., et al. 2000. Calcineurin expression, activation, and function in cardiac pressureoverload hypertrophy. Circulation. 101:2431-2437.

13. Lim, H.W., and Molkentin, J.D. 1999. Calcineurin and human heart failure. Nat. Med. 5:246-247.

14. Fatkin, D., et al. 2000. An abnormal $\mathrm{Ca}^{2+}$ response in mutant sarcomere protein-mediated familial hypertrophic cardiomyopathy. J. Clin. Invest. 106:1351-1359.

15. Moravec, C.S., Ruhe, S., Cifani, J.R., Milovanovic,
M., and Khairallah, P.A. 1994. Structural and functional consequences of minoxidil-induced cardiac hypertrophy. J. Pharmacol. Exp. Ther. 264:290-296.

16. Tsoporis, J., Fields, N., and Leenen, F.H. 1991. Contrasting effects of calcium antagonists vs. arterial vasodilators on cardiac anatomy and sympathetic activity in spontaneously hyper tensive rats. J. Cardiovasc. Pharmacol. 17:S166-S168.

17. Frey, N., McKinsey, T.A., and Olson, E.N. 2000 Decoding calcium signals involved in cardiac growth and function. Nat. Med. 6:1221-1227.

18. Olbrich, H.G., et al. 1993. Cyclosporine increases the intracellular free calcium concentration in electrically paced isolated rat cardiomyocytes. J. Heart Lung Transplant. 12:652-658.

19. Janssen, P.M.L., et al. 2000. Influence of cyclosporine A on contractile function, calcium handling, and energetics in isolated human and rabbit myocardium. Cardiovasc. Res. 47:99-107. 20. Park, K.S., Kim, T.K., and Kim, D.H. 1999 Cyclosporin A treatment alters characteristics of $\mathrm{Ca}^{2+}$-release channel in sarcoplasmic reticulum. Am. J. Physiol. 276:H865-H872.

21. Cameron, A.M., et al. 1995. Calcineurin associated with the inositol 1,4,5-trisphophate receptor-FKBP12 complex modulates $\mathrm{Ca}^{2+}$ flux. Cell. 83:463-472.

22. Schumann, K., Romanin, C., Baumgartner, W., and Groschner, K. 1997. Intracellular $\mathrm{Ca}^{2+}$ inhibits smooth muscle L-type $\mathrm{Ca}^{2+}$ channels by activation of protein phosphatase type $2 \mathrm{~B}$ and by direct interaction with the channel. J. Gen. Physiol. 110:503-513.

23. Gao, W.D., Perez, N.G., Seidman, C.E., Seidman, J.G., and Marban, E. 1999. Altered cardiac excitation-contraction coupling in mutant mice with familial hypertrophic cardiomyopathy. J. Clin. Invest. 103:661-666. 